\title{
Band-Gap Tunability in Partially Amorphous Silicon Nanoparticles Using Single-Dot Correlative Microscopy
}

\author{
Chia-Ching Huang,* Yingying Tang, Marco van der Laan, Jorik van de Groep, A. Femius Koenderink, \\ and Katerina Dohnalová*
}

Cite This: https://dx.doi.org/10.1021/acsanm.0c02395

Read Online

ACCESS |

山ll Metrics \& More

Article Recommendations

Supporting Information

ABSTRACT: Silicon nanoparticles (Si-NPs) represent one of many types of nanomaterials, where the origin of emission is difficult to assess due to a complex interplay between the core and surface chemistry. Band-gap tunability in Si-NPs is predicted to span from the infrared to the ultraviolet spectral range, which is rarely observed in practice. In this work, we directly assess the size dependence of the optical band gap using a single-dot correlative microscopy tool, where the size of the individual NPs is measured using atomic force microscopy (AFM) and the optical band gap is evaluated from single-dot photoluminescence measured on the very same NPs. We analyze $2-8 \mathrm{~nm}$ alkyl-capped Si-NPs prepared
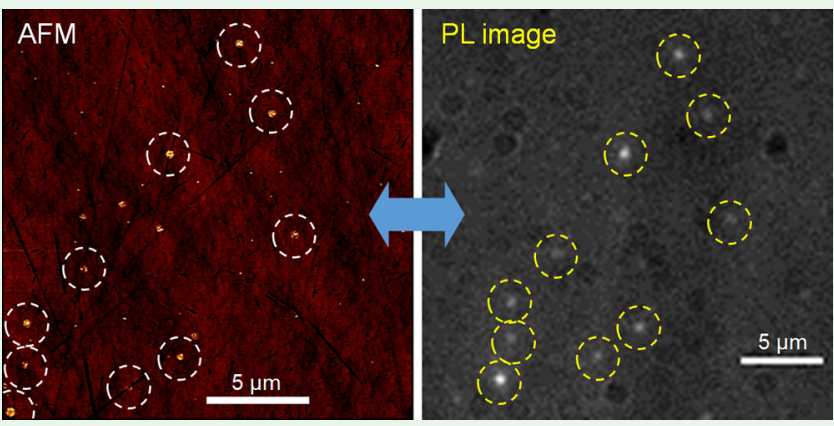
by a sol-gel method, followed by annealing at $1300{ }^{\circ} \mathrm{C}$.

Surprisingly, we find that the optical band gap is given by the amorphous shell, as evidenced by the convergence of the optical band gap size dependence toward the amorphous $\mathrm{Si}$ band gap of $\sim 1.56 \mathrm{eV}$. We propose that the structural disorder might be the reason behind the often reported limited emission tunability from various Si-NPs in the literature. We believe that our message points toward a pressing need for development and broader use of such direct correlative single-dot microscopy methods to avoid possible misinterpretations that could arise from attempts to recover size-band gap relation from ensemble methods, as practiced nowadays.

KEYWORDS: single-dot spectroscopy, correlative microscopy, silicon nanoparticles, size-tunability, amorphous silicon, quantum confinement

\section{INTRODUCTION}

Silicon ( $\mathrm{Si}$ ), an abundant and nontoxic material, is one of the most important semiconductors used nowadays. However, its indirect band gap renders its emission poor, which hampers its use in optoelectronic and photonic devices, such as lightemitting diodes or lasers. Also, its band-edge absorption is very inefficient, inhibiting the use of $\mathrm{Si}$ in thin-cell photovoltaics.

This is partially improved in small Si nanoparticles (Si-NPs) via quantum confinement effect (QCE) and surface engineering. ${ }^{1-3}$ Similar to other NPs, Si-NPs with a radius smaller than the excitonic Bohr radius have size-tunable band-gap energy. $^{2,4-9}$ According to the effective mass approximation (EMA), the band-gap energy of a spherical crystalline NP deviates from its bulk value in inverse proportion to the square of the NP's radius (the localization term) plus the term that scales inverse with the radius (polarization term), ${ }^{10}$ and a similar trend is also predicted by the first-principles methods. ${ }^{11,12}$ Theoretical predictions show that the emission tunability range of the Si-NPs should be one of the broadest among all of the semiconductors, spanning from the nearinfrared (NIR) spectral range at $1.1 \mathrm{eV}$ to the ultraviolet (UV) range at over $3.5 \mathrm{eV} .^{2,6,8,9} \mathrm{In}$ practice, however, weaker and/or limited size dependencies are observed. ${ }^{2,9}$ Such deviations from the predicted trend are often discussed in terms of the presence of extrinsic radiative channels, such as localized surface states or as a result of strain. ${ }^{2,13-15}$

In this work, we employ a direct experimental method for assessing the size dependence of the band-gap energy by a custom-built single-dot correlative microscopy tool combining optical single-dot microspectroscopy with atomic force microscopy (AFM). We use this tool to analyze the origin of emission from sol-gel-synthesized alkyl-capped Si-NPs. Such a material was earlier reported to consist of a crystalline Si core and an amorphous Si shell, despite annealing at $1300{ }^{\circ} \mathrm{C}$, as evidenced by a detailed nuclear magnetic resonance (NMR) study. ${ }^{16}$ In their follow-up study, the authors concluded that the optical properties are dictated by the crystalline core, ${ }^{17}$

Received: September 28, 2020

Accepted: December 4, 2020 
based on an indirect, ensemble optical properties and X-ray diffraction (XRD) size analysis. We do confirm that such a material is partially amorphous, but using our direct size-band gap measurement, we find that the band gap for larger Si-NPs converges to $1.59 \mathrm{eV}$, which is the bulk band gap of amorphous Si. Our result shows that ensemble analysis can be misleading and that there is a critical need for such direct correlative measurements to be implemented in assessment of emission origin, especially for complex materials such as Si-NPs. Also, we suggest that disorder might be another often-neglected reason for the observed limited size-tunability in Si-NPs and similar materials.

\section{RESULTS AND DISCUSSION}

The structure and synthesis of the Si-NPs analyzed in this work are schematically summarized in Figure 1. Si-NPs were
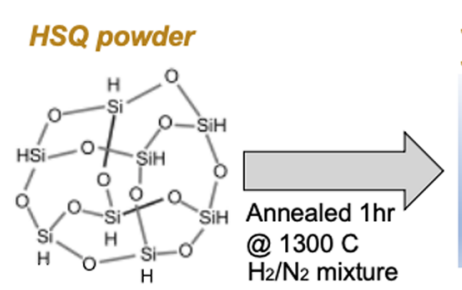

Si-NPs inside

$\mathrm{SiO}_{2}$ matrix

$\mathrm{H}_{2} / \mathrm{N}_{2}$ mixture

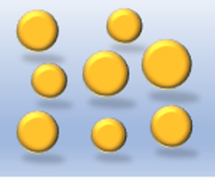

Etching in

HF/water (1:1)
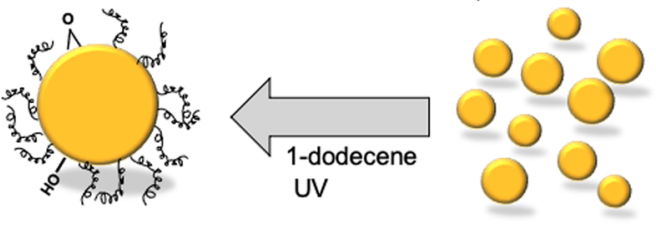

freestanding

Alkyl-capped

Si-NPs
PLE and extinction spectra. This is in agreement with the observed lack of aggregation over several years of storage of the Si-NPs in toluene in the dark. The absorption spectrum is featureless, as expected for an indirect band-gap semiconductor material, with no prominent band-gap edge. To evaluate the band-gap edge, we plot absorption spectra in the Tauc plot for the indirect allowed transition (Figure S2), which reveals an optical band gap of $2.21 \pm 0.01 \mathrm{eV}$. Steady-state PL spectra excited at three different wavelengths by a low-power continuous-wave (cw) laser peak close to $813 \mathrm{~nm}$ (inset in Figure S1). The full PLE map reveals only a negligible spectral shift of $\sim 20 \mathrm{meV}$ of the PL peak with the excitation wavelength (Figure S3), in agreement with the indirect band-gap character of the material and the resulting large Stokes shift between the excitation and emission energies. PL quantum yield (QY) was measured on this sample previously ${ }^{19}$ and found to be above $40 \%$. This is a very high value for such Si-NPs, making them good a material for the demonstration of correlative single-dot spectroscopy here. Similarly, high QY values for this type of material have also been previously reported by other groups. ${ }^{20,21}$

Due to the size-effect, every single Si-NP has a different band gap and a radiative rate. In such a case, the ensemble optical analysis offers only a summed-up PL spectrum, whose peak does not directly translate into the mean Si-NP size. The emission rate of the single Si-NP depends strongly on its size, owing to the size-dependent radiative rate. Hence, the smaller Si-NPs are much brighter and contribute more to the ensemble PL than the larger Si-NPs. This means that the mean PL peak does not coincide with the mean Si-NP size but is blue-shifted. Furthermore, defect states (e.g., from silica oxide ${ }^{2,6,22-24}$ ) can become stabilized inside the band gap in the smaller Si-NPs (and not the larger ones), owing to the band-gap opening. At the same time, smaller Si-NPs might be more prone to disorder and sensitive to the environment. Hence, the size dependence of the optical band gap of NPs cannot be inferred from the ensemble optical properties and requires in-depth single-NP PL spectroscopy, correlated with a size analysis of the same NPs. This can be done using, for example, our setup, described in Figure 2a (more details can be found in the Methods section), which allows us to directly measure and correlate for each single Si-NP its size, obtained from the AFM scan of the surface topography and measured with sub-nanometer resolution, with its optical band gap, obtained from the analysis of the single Si-NP PL spectrum. The procedure is sketched in Figure $2 \mathrm{~b}-\mathrm{e}$. First, a PL image of a large $25 \times 25$ $\mu \mathrm{m}^{2}$ area (=field of view (FOV)) is visualized by an optical microscope (Figure 2b) to identify the "anchoring" emissive nano-objects, such as the single Si-NPs or their clusters. This initial PL imaging is done very briefly (with an acquisition time of $8 \mathrm{~s}$ ) to avoid photobleaching prior to the spectral analysis. The same area is consequently slowly scanned by an atomic force microscope in a tapping mode (Figure 2b) and the two images are correlated using the large anchors (encircled in Figure $2 b, c$ ). The tapping mode is used to avoid strong tipsample interactions that could lead to displacement or removal of the Si-NPs during scanning. Afterward, the whole area is rescanned with a narrow slit placed in the image plane, using a longer acquisition time of $6 \mathrm{~min}$ for each spectrum. This allows us to capture PL spectra of all of the nano-objects in the scanned area and also those invisible to the initial PL image scan used for correlative purposes only. In Figure $2 \mathrm{~d}$,e, an example of a PL spectrum and size obtained from a Si-NP that 

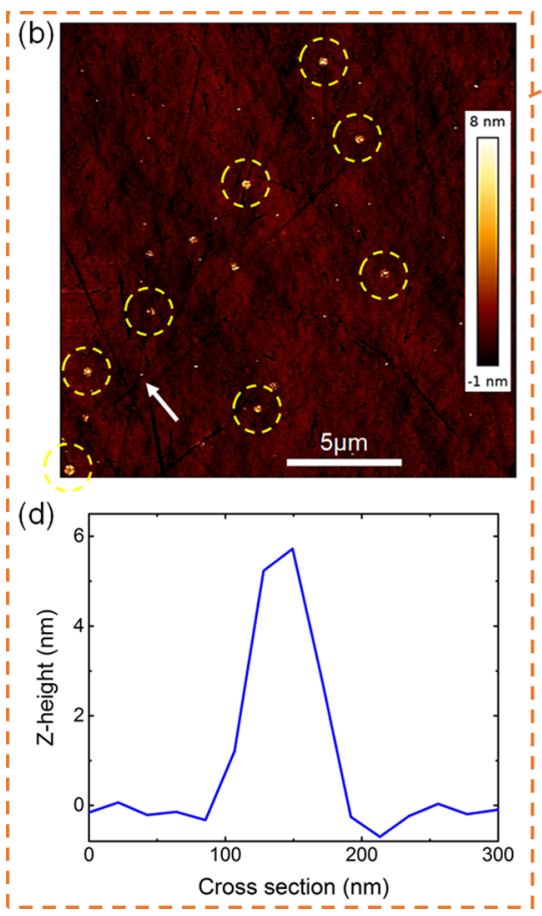

(a)

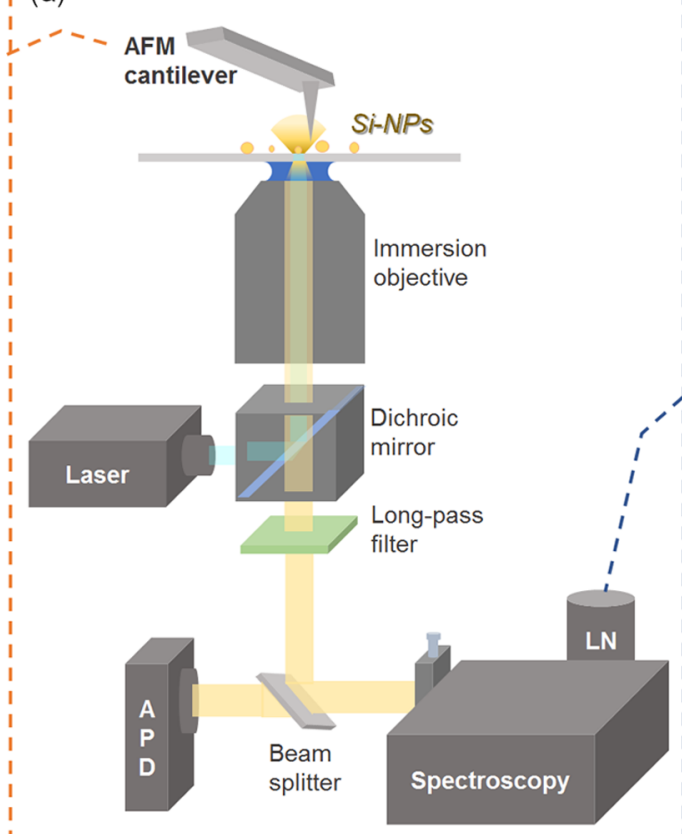

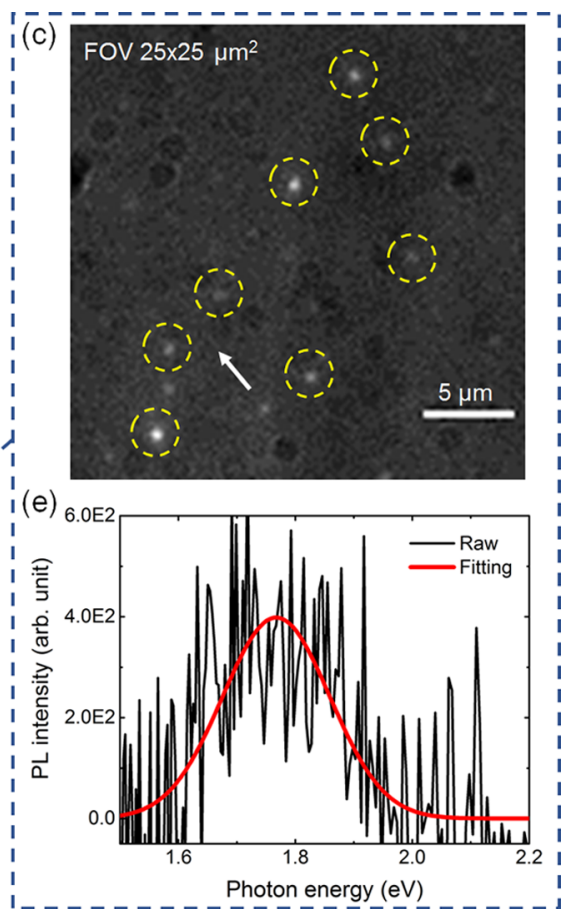

Figure 2. (a) Schematic of the correlative microscope setup comprising an inverted optical microscope and an atomic force microscope. The microscope is coupled via a beam splitter to an avalanche photodiode (APD) and connected to a photon-correlating card and a spectrometer with a CCD detector cooled by liquid nitrogen (LN). (b) An example of an AFM scan image of the sparsely deposited Si-NPs on a high-quality quartz substrate. The scale bar is $5 \mu \mathrm{m}$. (c) PL microscopy image of the same area as in (b), acquired by the CCD detector. The PL image shown is a cutout of the whole field of view (FOV), which is $25 \times 25 \mu \mathrm{m}^{2}$. The anchoring Si-NPs used for correlation purposes are encircled using yellow dashed circles. The whole scanned area is rescanned with a narrow horizontal slit in the image plane and longer acquisition times to capture the single Si-NP PL spectra. This often reveals small Si-NPs that were invisible in the initial PL scan. This is also the case of a Si-NP indicated by an arrow in (b) and (c), whose PL spectrum and size are shown in (e) and (d), respectively.

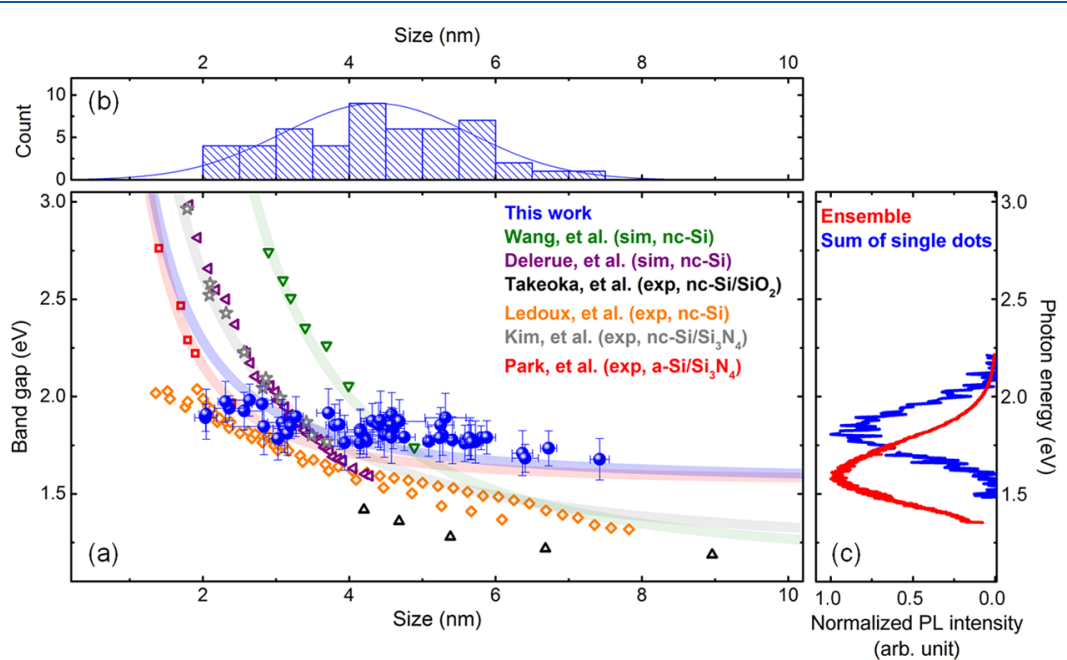

Figure 3. (a) Plot of the optical band gaps for the likely single Si-NPs (blue spheres) obtained from fitting the PL peak of 50 single Si-NPs spectra as a function of the Si-NPs size evaluated from the AFM measurements. The vertical "error bars" are given as the FWHM of the single-dot PL spectra when fitted by a single Gaussian peak. The horizontal "error bar" is given by the average substrate roughness, which is a good estimate for the uncertainty in the measured single Si-NP height. Experimental data from this study (blue spheres) are compared with the literature: theoretical simulations of crystalline Si-NP by Wang et al. ${ }^{27}$ (green inverted triangle) and by Delerue et al. ${ }^{28}$ (purple inverted triangle) and experimental results on crystalline Si-NPs in $\mathrm{SiO}_{2}$ matrix prepared by Takeoka et al. ${ }^{29}$ (black triangle), crystalline Si-NPs by Ledoux et al. ${ }^{30}$ (orange diamond), crystalline Si-NPs in $\mathrm{Si}_{3} \mathrm{~N}_{4}$ matrix by Kim et al. ${ }^{31}$ (gray star), and amorphous Si-NPs in $\mathrm{Si}_{3} \mathrm{~N}_{4}$ matrix by Park et al. ${ }^{32}$ (red square). Thick semitransparent lines are EMA model fits using fitting parameters reported by Wang et al. ${ }^{27}$ (green inverted triangle), Park et al. ${ }^{32}$ (red square), and Kim et al. ${ }^{31}$ (gray star). The blue line represents our own fit using the EMA model. (b) Histogram of the single Si-NP sizes, as obtained from the AFM topography scans, indicates a broad size distribution with a mean size of $4.4 \pm 1.3 \mathrm{~nm}$. (c) Ensemble PL spectrum (red line) compared with the sum of the 50 selected single-dot PL spectra (blue line), both measured in the same microscopy setup here with excitation by a $445 \mathrm{~nm}$ diode laser in the cw regime. PL peaks from the sum of single Si-NPs and ensemble are at 1.82 and $1.53 \mathrm{eV}$, respectively. 

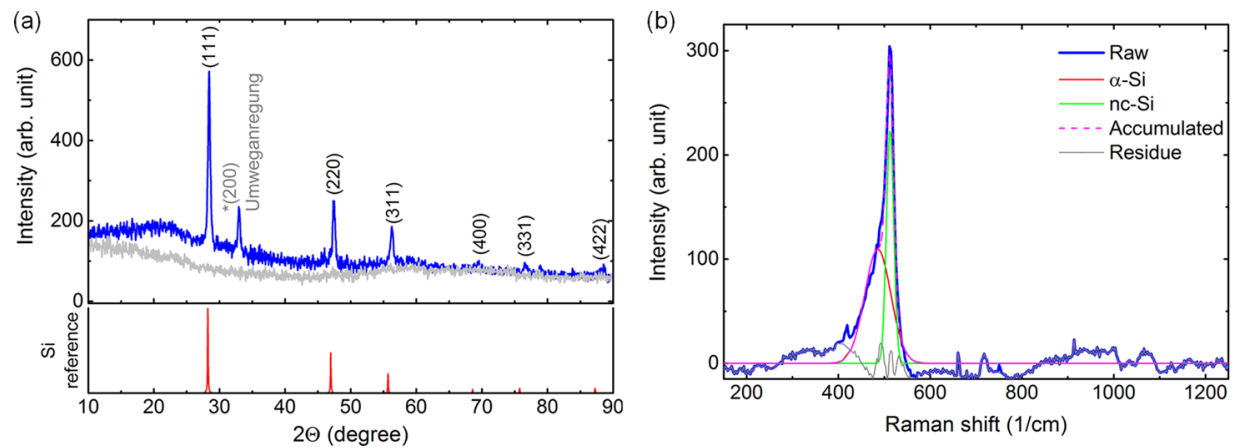

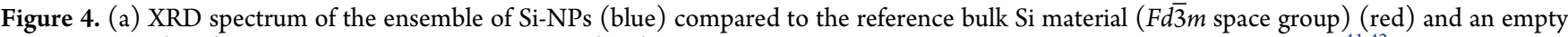
sample holder (gray). The peak denoted by an asterisk (200) is a forbidden reflection that can arise from multiple diffractions, ${ }^{41,42}$ distortion of the $\mathrm{Si}$ lattice, ${ }^{43}$ or Bragg reflection of the $\mathrm{Cu} \mathrm{K} \alpha$ radiation. ${ }^{44}$ (b) Raman spectrum of one of the emissive Si-NP clusters (blue line) fitted by two Gaussian components at $485.9 \mathrm{~cm}^{-1}$, corresponding to the amorphous Si phase, and $512.7 \mathrm{~cm}^{-1}$, corresponding to the nanocrystalline Si phase, shifted from the bulk $\mathrm{Si}$ crystalline peak at $520 \mathrm{~cm}^{-1}$.

was initially not visible (VIS) on the brief PL scan (position is indicated by the arrow in Figure $2 b, c)$ is shown. PL spectra of all of the Si-NPs within the scanned area are recorded without any selectivity by the spectrometer and a charge-coupled device (CCD), fitted and matched with the height information from the AFM scan, using the "anchoring" clusters. In this way, we obtain a direct measurement of the size dependence of the optical band gap of the Si-NPs. Since the lateral resolution of the atomic force microscope is limited by the size of the tip (usually larger than $10 \mathrm{~nm}$ ), it is very difficult to prove that there is only a single Si-NP without including additional measurements, such as antibunching. We note that this is technically possible in our setup but was not used for these $\mathrm{Si}$ NPs because of their slow PL lifetime. Hence, to make sure that our data are likely from single Si-NPs, we include for the final analysis of the size-band gap relation only the PL spectra with the narrowest full width at half-maximum (FWHM) and the lowest PL intensity within the respective spectral range, with the assumption that aggregates of multiple emissive SiNPs of similar sizes effectively multiply the integral PL signal.

Our main results are summarized in Figure 3 that shows (a) an overview of the optical band gaps as a function of the NP size for all of the 50 measured likely single emitting Si-NPs (blue circles). Optical band gaps are approximated as the peak of each single-NP PL spectrum when fitted to an energy scale by a single Gaussian peak function (Figure S4) to reflect the fact that the measured PL spectrum originates from multiple transitions over time from a variety of states that are populated at room temperature (as opposed to a single transition that would lead to a Lorentzian shape). The PL spectra might consist of phonon-related replicas and be influenced by spectral diffusion, ${ }^{25}$ but given the noisy PL signal, this is hard to define. Nevertheless, we expect that a possible phonon structure and/or spectral diffusion can introduce error in the band-gap evaluation by only less than $100 \mathrm{meV}$, which does not influence critically the main size-band gap tunability result. A histogram of the fitted FWHMs of all of the evaluated single-NP PL spectra is plotted in Figure S5, revealing a broad mean FWHM of $200 \mathrm{meV}$, which is in excellent agreement with earlier independent measurements of a similar material by another group. ${ }^{26}$ Sizes of the respective Si-NPs are retrieved from the topography profile measured by the atomic force microscope in tapping mode. The resolution of this analysis is a sub-nanometer for the height, limited by the mean roughness of the substrate, which is found to be below $0.3 \mathrm{~nm}$ (Figure
S9). This is true only for the fresh high-quality quartz substrates, whose surface is found to generally become rougher with usage and repeated cleaning. The histogram of the Si-NP sizes obtained by AFM is shown in Figure $3 \mathrm{~b}$ and gives a mean size of $4.4 \pm 1.3 \mathrm{~nm}$ for the optically analyzed Si-NPs.

According to the effective mass approximation (EMA) model, the dependence of the optical band gap $E_{\mathrm{g}}(R)$ on the radius of the NP $R$ can be fitted by the Brus equation ${ }^{10}$

$$
E_{\mathrm{g}}(R) \simeq E_{\mathrm{g}}(\infty)+\frac{\hbar^{2} \pi^{2}}{2 R^{2}}\left[\frac{1}{m_{\mathrm{e}}}+\frac{1}{m_{\mathrm{h}}}\right]-\frac{1.8 \mathrm{e}^{2}}{\epsilon R}
$$

where $E_{\mathrm{g}}(\infty)$ is the band-gap energy of the bulk material at room temperature, $\hbar$ is reduced Planck constant, $m_{\mathrm{e}}$ and $m_{\mathrm{h}}$ are the effective masses of the electron and hole quasi-particles, respectively, and $\epsilon$ is the permittivity. The localization $1 / R^{2}$ term for NPs in a strong confinement regime is much larger

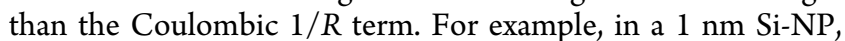
the Coulombic term is only about $120 \mathrm{meV}^{33}$ while the localization term reaches few electronvolts. Therefore, it is usually considered that $E_{\mathrm{g}}(R) \propto 1 / R^{x}$, where $1<x<2$. Data by Park et al., ${ }^{32}$ Kim et al., ${ }^{3}$ and Wang et al. ${ }^{27}$ are fitted by EMA model parameters described in their respective studies with the results shown in Figure $3 \mathrm{a}$ in red, gray, and green thick semitransparent lines, respectively. Comparing our measured size dependence of the optical band gap in Figure 3a, we find a clear mismatch with all of the size dependencies theoretically simulated or experimentally measured for crystalline Si-NPs, especially for the larger Si-NP sizes. Instead, our result follows data reported experimentally for the amorphous Si-NPs by Park et al., ${ }^{32}$ fitted with a fixed $h^{2} / 2 m^{*}=2.39$, a bulk band gap of $1.56 \mathrm{eV}$, and a power coefficient of $x \sim 1.72$ (blue line in Figure 3a), characteristic for a bulk amorphous $\mathrm{Si}^{34}$ Hence, our results strongly suggest that the analyzed Si-NPs are either fully amorphous or that the PL is dominated by the amorphous part of the Si-NP. This is a very interesting result, especially considering a recent study on a similar material system ${ }^{17}$ that suggests that it is the crystalline part that dominates the PL.

To characterize the crystallinity of our material, we performed X-ray diffraction (XRD) on the ensemble of SiNPs and Raman microspectroscopy on a cluster of emissive SiNPs (Figure 4). The XRD spectrum exhibits a characteristic diamond structure of crystalline $\mathrm{Si}$ with signals from characteristic (111), (220), (311), (400), (331), and (422) planes, confirming the presence of a crystalline Si phase in the Si-NP 


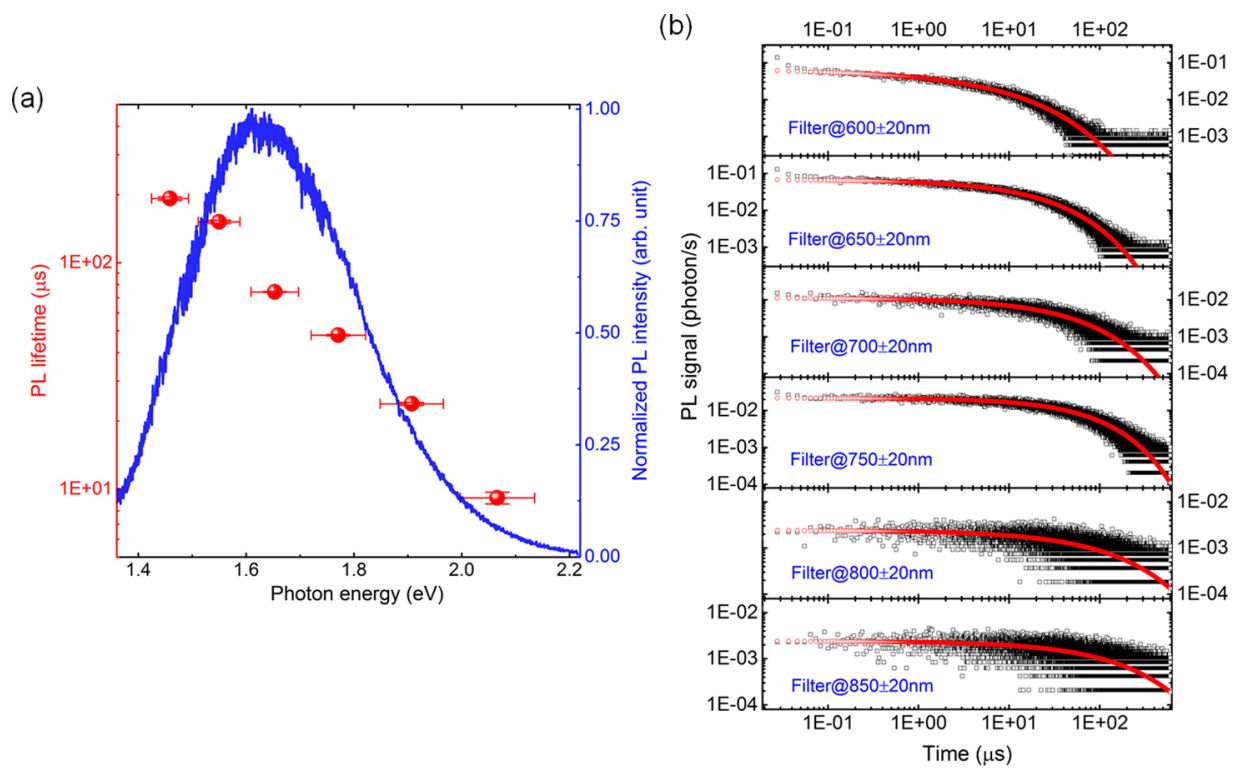

Figure 5. (a) Ensemble PL spectrum (blue line; $y$-axis on the right-hand side) of the Si-NP sample and PL lifetimes (red sphere; $y$-axis on the lefthand side) measured using band-pass filters $(40 \mathrm{~nm}$ width) centered at $600 \mathrm{~nm}(2.07 \mathrm{eV}), 650 \mathrm{~nm}(1.91 \mathrm{eV}), 700 \mathrm{~nm}(1.77 \mathrm{eV}), 750 \mathrm{~nm}(1.65$ $\mathrm{eV}), 800 \mathrm{~nm}(1.55 \mathrm{eV})$, and $850 \mathrm{~nm}(1.46 \mathrm{eV})$. (b) PL lifetimes (black symbols) measured using the band-pass filters fitted by a stretched exponential function (red symbol).

structure. The signal is compared to a reference bulk crystalline Si material and an empty sample holder. The broad signature of the background results from the amorphous $\mathrm{Si}$ (see also Figure S12 for the sample XRD with the subtracted background), as it closely follows the signal detected from the sample holder (gray line). ${ }^{35}$

The Raman spectra of several brightly emissive Si-NP clusters were measured using a Raman microscopy system (Figure $4 \mathrm{~b}$ and for the other two clusters, Figure S11). The Raman signal shows two main components: (i) a strong narrow component (green) at $512.7 \mathrm{~cm}^{-1}$ and a broad peak at around $486 \mathrm{~cm}^{-1}$. The first peak corresponds to the welldocumented nanocrystalline $\mathrm{Si}^{36-38}$ which is strongly redshifted with respect to the bulk crystalline $\mathrm{Si}$ signal at 521 $\mathrm{cm}^{-1}$. The broader signal at $486 \mathrm{~cm}^{-1}$ corresponds to amorphous $\mathrm{Si}^{37,38}$ confirming the presence of both crystalline and amorphous phases within the Si-NPs. We also confirm that the sample is oxide-free, since we observe no features related to silica oxide $\mathrm{e}^{39,40}$ in any of the Raman spectra. The presence of $\mathrm{Si}-\mathrm{O}$ bonds on the $\mathrm{Si}-\mathrm{NP}$ surface in small quantities is expected, due to the fact that surface coverage by organic ligands can only be partial due to steric hindrance. Nevertheless, the excellent colloidal stability of these Si-NPs in toluene and hexane indicates that the majority of the surface is organically coated. In summary, the Si-NPs are not fully oxidized nor fully amorphous but contain both amorphous and crystalline phases, likely in a form of a core-shell system, as suggested in the study by Veinot et al. ${ }^{16}$ Therefore, our results showing the convergence of the size-dependent band gap toward the bulk amorphous $\mathrm{Si}$ band gap of $1.59 \mathrm{eV}$ in Figure 3 a suggests that it is the amorphous phase that dictates the optical properties. We note here that besides the indication of crystallinity, XRD and Raman spectra can also be used to obtain an estimate of the NP mean size (see Supporting Information); however, such an evaluation is not without a considerable error of evaluation of the size of a crystalline core-amorphous shell system.
Further support for a strong involvement of the disordered phase in the PL process is provided by the fact that the singleNP PL spectral widths, quantified by the FWHM evaluated from the fitting by a Gaussian function (Figure S4), are very broad, about $200 \mathrm{meV}$ in average (Figure S5). A high amount of disorder leads to the broadening of the phonon spectra, which in turn broadens the homogeneous line width of the single-dot PL spectrum. An alternative interpretation of the broad single-dot spectra observed in a material of a similar origin was put forward by Sychugov et al., ${ }^{26}$ who argued that the phonon mode energies are strongly broadened in the softorganic-shell-capped Si-NPs with respect to the hard-oxideshell-coated Si-NPs. As a result, the breathing mode of the strong lowest-order NP splits into several directional modes, with further broadening arising from shape irregularities and strong size dependence of the acoustic phonon modes. However, we believe that a possible amorphous shell presence within these Si-NPs likely leads to a similar output and is therefore an alternative interpretation of the strongly broadened single-NP PL spectra confirmed here. An additional reason for wide single-dot $\mathrm{PL}$ spectra can be spectral diffusion, ${ }^{25}$ which could introduce an additional error in the band-gap evaluation of the order of $50-100 \mathrm{meV}$. This is, however, impossible to define under the given ambient experimental conditions.

It is interesting to realize that the size of Si-NPs estimated by STEM (Figure S10) is larger than the mean size obtained for the 50 emissive Si-NPs in Figure $3 \mathrm{~b}$. There are three factors that need to be discussed. First, in ambient condition AFM measurements, the omnipresent water thin film causes strong attractive capillary forces onto the tip, ${ }^{45}$ which shifts the oscillation frequency of the tip. These capillary forces are reduced above the NP, which in the end makes the NP appear smaller than it really is. ${ }^{45}$ Second, one might expect that the ligand contributes to the height, since the extended dodecene molecule is $\sim 1.67 \mathrm{~nm}$ long, ${ }^{46}$ but this is in fact a negligible effect, $^{45,47}$ owing to the strong surface lateral capillary forces and low degree of surface coverage by the alkyl chains limited 
by steric hindrance. Third, and most importantly, it needs to be noted that the AFM histogram in Figure $3 \mathrm{~b}$ does not represent the Si-NP ensemble, like the STEM size evaluation, but only the $50 \mathrm{Si}-\mathrm{NPs}$ that were selected through the singleNP PL spectroscopy. This is only a small subset of the Si-NP ensemble. Single-dot spectroscopy is known to be systematically biased toward the brighter and hence smaller Si-NPs, ${ }^{48,49}$ owing to their size-dependent radiative rate. To compare the PL of the analyzed subset of the $50 \mathrm{Si}$-NPs with the Si-NP ensemble, we sum up all of the single-NP PL spectra and compare the result with the NP ensemble PL spectrum, excited and measured under the same experimental conditions (Figure $3 c$ ). We find a blue shift in the summed-up single-NP PL spectrum, with respect to the ensemble PL spectrum, which agrees with the shift of the size histogram toward smaller SiNPs.

The stronger contribution of the smaller Si-NPs can be quantitatively analyzed by measurement of the spectrally resolved PL lifetime. In Figure 5a, we show spectrally resolved average PL lifetimes $\langle\tau\rangle$ measured under a picosecond-pulsed excitation at $445 \mathrm{~nm}$ and plotted against the PL ensemble spectrum. The spectral resolution is achieved by the use of narrow (40 nm bandwidth) band-pass filters in the detection chain. The as measured time-resolved PL signal $I(t)$ is shown in Figure $5 b$, fitted by a stretched exponential function $I(t) \propto \exp \left[-\left(t / \tau_{\mathrm{K}}\right)^{\beta}\right]$ and yielding an average lifetime $\langle\tau\rangle=$ $\left(\tau_{\mathrm{K}} / \beta\right) \Gamma(1 / \beta)$, depicted in Figure 5a (red circles). The ensemble results without spectral filtering are shown for comparison in Figure S6. As expected, the average PL lifetimes show a strong spectral dependence, as a result of size-effect in indirect band-gap Si-NPs. In particular, we found the average PL lifetime within the spectral band of $600 \pm 20 \mathrm{~nm}$ to be 9.1 $\mu \mathrm{s}$, increasing gradually up to $192 \mu \mathrm{s}$ for the $850 \pm 20 \mathrm{~nm}$ spectral window, suggesting that the smaller Si-NP would appear approximately 20 times brighter than the larger Si-NP, assuming that they are subjected to the same nonradiative channels. Smaller Si-NPs are therefore more visible in the single-NP PL spectroscopy used for selecting the Si-NPs for our correlative study.

The notion that emission is likely dominated by the amorphous parts of the Si-NP structure might be surprising because these Si-NPs were annealed up to $1300{ }^{\circ} \mathrm{C}$, which is usually considered high enough to induce full crystallization. However, recent research by several groups indicates that the assumption of full crystallization upon high-temperature annealing might be flawed. First, experimental results by Thiessen et al. ${ }^{16}$ indicate that Si-NPs annealed to $1300{ }^{\circ} \mathrm{C}$ are composed of a crystalline core and an amorphous shell. This was shown experimentally using $\mathrm{Si}(29)$ solid-state nuclear magnetic resonance (NMR) spectroscopy, probing the surface of large amounts of hydrogen-terminated Si-NPs ranging from 3 to $64 \mathrm{~nm}$ in size. Another recent experimental study by Alessi et al. ${ }^{50}$ on plasma-synthesized $\mathrm{Si}$-NPs suggested that the crystallinity of the Si-NP of the same size can be manipulated by changing the concentration of silane, which changes optical and electronic properties of the material. More importantly, a recent theoretical study by Talyzin et al. ${ }^{51}$ suggests that molten $\mathrm{Si}$ nanodroplets do not return to the crystalline form but remain fully amorphous and that Si-NP surface has a melting temperature different from that of its core. The phaseequilibrium model of crystallization indicates that the melting temperature of NPs decreases relative to the bulk melting temperature $\left(\sim 1414{ }^{\circ} \mathrm{C}\right.$ for $\left.\mathrm{Si}\right)$ linearly with their reciprocal radius $T_{\mathrm{m}}\left(R^{-1}\right),{ }^{52,53}$ though this model does not work very well for covalent materials like $\mathrm{Si}$, where formation of defects (and hence also surfaces) has dynamics different from that in the simpler ionic/metallic nanosystems. This is more appropriately treated in the so-called surface-phonon instability model, ${ }^{54}$ accounting for the lowering energy of the phonon modes with increasing concentration of defects, offering a better fit to the experimentally observed data. ${ }^{5,56}$ These observations indicate that melting of the disordered surfaces occurs at lower temperatures compared to the melting of the crystalline core, in agreement with the aforementioned study by Talyzin et al. ${ }^{51}$ This could rationalize the formation of a crystalline coreamorphous shell Si-NP system.

It is important to also discuss the recent study by Thiessen et al., ${ }^{17}$ which puts forward an opposing interpretation of the PL from such crystalline core-amorphous shell Si-NP systems. In their study, the authors measure an average Si-NP size using XRD and transmission electron microscopy (TEM) and ensemble optical properties, showing that Si-NP batches with the same mean outer size, but with various crystallinities, show very different PL spectra and lifetimes. It is argued that such results indicate that it is the crystalline core, and not the amorphous shell, that dominates the optical properties. However, we would like to note that ref 17 only offers an indirect correlation between the optical band gap and size and that the authors choose to fit the size dependence of both samples with a fixed bulk band-gap value, the crystalline $\mathrm{Si}$ one, instead of fitting the band gap to their data. Hence, we see controversy only in the interpretation and data analysis and not in the data itself, which should be further scrutinized.

\section{CONCLUSIONS}

In summary, we show a direct measurement of the size dependence of the band gap in the Si-NP system that has been previously reported to contain a crystalline core and an amorphous shell, ${ }^{16}$ with the core dominating the PL. ${ }^{17}$ The measured size-dependent PL band gap is fitted using the Brus equation and found to better fit the amorphous bulk Si band gap than the crystalline bulk Si one. This is very interesting for several reasons.

(i) First, it is somewhat surprising that annealing at $1300{ }^{\circ} \mathrm{C}$ does not render the Si-NPs fully crystalline. It has been assumed for a long time that NPs require a temperature lower than the bulk melting temperature to melt and it was assumed that upon cooling of a fully melted $\mathrm{Si}$ nanodroplet, one would obtain fully crystalline Si-NPs. However, this assumption is challenged by a recent study by Talyzin et al. ${ }^{51}$ that shows that melted nanodroplets of Si might in fact never return to their crystalline form and that the surface of Si-NPs melts faster than their core, which could result in crystalline core-amorphous shell Si-NP systems. This is in agreement with our observations and two other studies by Thiessen et al. ${ }^{16,17}$ In fact, most studies in the literature do not exceed annealing over $1300{ }^{\circ} \mathrm{C}$ and do not pay special attention to the requirements of very slow cooling rates. ${ }^{51}$ This could mean that many materials earlier presented as crystalline Si-NPs might have been partially or fully amorphous, where the relatively thin amorphous $\mathrm{Si}$ shell would have been difficult to identify via XRD, Raman spectroscopy, or other means. 
(ii) Second, another study on a similar material by Thiessen et al. ${ }^{17}$ arrived at an opposite interpretation that the PL is dominated by the crystalline $\mathrm{Si}$ core. However, their analysis is only indirect, and data were fitted assuming convergence to a bulk $\mathrm{Si}$ band gap. Therefore, we suggest that there is a critical need for direct sizedependence band-gap measurements by correlative single-dot methods, as performed here. Also, we suggest that the band gap of the material should not be assumed but fitted.

(iii) Third, sol-gel synthesis has so far resulted in the most promising emission efficiencies with a high QY of $40 \%$ in the red spectral range for oxide-free Si-NPs. ${ }^{19}$ This synthesis is easily scalable and hence interesting for industrial applications. However, unlike most organically capped Si-NPs, this material exhibits a slow PL lifetime, very broad single-dot PL spectra, and a red-shifted PL with a weak size dependence, typically attributed to oxide-capped Si-NPs. In our study, we confirm that this material is not oxidized and contains both crystalline and amorphous Si. However, it seems that the amorphous shell dominates the PL and hence leads to a weaker size dependence of the band gap. Hence, we would like to suggest that an often observed weak size dependence of the optical band gap in Si-NPs in the literature might also be attributed to the presence of the disordered phase.

\section{METHODS}

Synthesis of Si-NPs was carried out by the group of Veinot and is described in detail elsewhere. ${ }^{18}$ In brief, Si-NPs were prepared from hydrogen silsesquioxane (HSQ), annealed in an atmosphere of $5 \%$ hydrogen $\left(\mathrm{H}_{2}\right)$ and $95 \%$ argon $(\mathrm{Ar})$ for $1 \mathrm{~h}$ at $1300{ }^{\circ} \mathrm{C}$. The process leads to substoichiometric silica $\mathrm{SiO}_{x}$, which after annealing forms $\mathrm{Si}$ NPs inside a stoichiometric silica $\left(\mathrm{SiO}_{2}\right)$ powder. To release the $\mathrm{Si}$ NPs, the powder is dispersed in a solution of hydrogen fluoride (HF) and deionized water with a ratio of $1: 1$. Then, the Si-NPs are dissolved in toluene and 1-dodecene mixtures, where 1-dodecene encapsulates the Si-NP by photochemical hydrosilylation, initialized under $365 \mathrm{~nm}$ UV illumination. Afterward, centrifugation is used to separate the Si-NPs from the solvent, and Si-NPs are redispersed in toluene. The schematic sketch of the synthesis and the final Si-NP is shown in Figure 1.

For the optical spectroscopy of the Si-NP ensemble, Si-NPs are kept in solution, dispersed in toluene, and placed inside quartz cuvettes (110-QS from Hellma-Analytics). For the correlative singledot microspectroscopy and AFM measurements, we dilute the Si-NP solution with hexane ( $\geq 99.5 \%$ purity, Sigma-Aldrich) and deposit it on a $165 \mu \mathrm{m}$ thick quartz slide (SPI Supplies) with an approximate deposition density of $\sim 0.08$ particle $/ \mu \mathrm{m}^{2}$. Slides are cleaned beforehand first by a mixture of Hellmanex III (Sigma-Aldrich) with $0.5-2 \%$ weight concentration in pure water from Merck Milli-Q water purification system at $40{ }^{\circ} \mathrm{C}$ for $25 \mathrm{~min}$ and finally washed in pure water (Merck Milli-Q) for $10 \mathrm{~min}$. This procedure creates a hydrophilic surface on the coverslip, which allows for homogeneous deposition of the Si-NPs with a selective solvent for single-dot microspectroscopy. The cleaning method was tested for its effects on the average roughness of the coverslip, which is found to stay below $0.3 \mathrm{~nm}$ (Figure S9). This is an important prerequisite for a highresolution AFM analysis of the Si-NPs. Quartz slides with a refractive index of $n=1.56$ at $400 \mathrm{~nm}(3.100 \mathrm{eV})$ are selected for their high transmittance $T=0.93$ in the visible (VIS) spectral range and almost no emission from the usually expected surface point defects, such as nonrelaxed oxygen vacancies. This allows us to measure Si-NPs's single-dot spectra despite their broad emission spectra and low radiative rate, both resulting in a low detected signal. The signal-to- noise ratio is improved using long acquisition times for both the single-dot PL images and spectra.

The optical microscopy system comprises an inverted microscope (Zeiss Axio Observer Z1) where the original Zeiss mechanical sample stage is replaced by a stabilized nanoprecision tip-assisted optical scanning stage (TAO Nanowizard 3; JPK Instruments). The microscope is equipped with an oil-immersion objective (Zeiss; Plan-Apochromat, $100 \times$ magnification, $\mathrm{NA}=1.4$ ) and a filter cube with a dichroic mirror (Semrock; 442-nm laser BrightLine) and a long-pass filter (Semrock; 458-nm EdgeBasic). Excitation is performed by a $445 \mathrm{~nm}$ solid-state diode laser (Becker \& Hickl $\mathrm{GmbH}$; BDL-445-SMN) operating either in a continuous wave (cw) mode (maximum average power of $7.40 \mathrm{~mW}$ ) or a pulsed mode $(9.2$ ns pulses with a $1428.6 \mathrm{~Hz}$ repetition rate). The PL spectra were excited by a round approximate Gaussian profile excitation laser spot with a radius of $\sim 40 \mu \mathrm{m}$ and an average power of $6.28 \mathrm{~mW}$; hence, the excitation density is estimated to be $\sim 130 \mathrm{~W} / \mathrm{cm}^{2}$. To detect spectrally resolved PL spectra of single NPs, we use a spectrometer (Acton SP2300; Princeton Instruments; grating with 300 grooves/ $\mathrm{mm}$, blazed at $750 \mathrm{~nm}$ ) coupled to a Si-based liquid nitrogen (LN)cooled charge-coupled-device (CCD) detector (Pylon 400B) array with $1340 \times 400$ pixels and a pixel size of $20 \times 20 \mu \mathrm{m}^{2}$. To measure the PL lifetime, we use an avalanche photodiode (APD; IDQuantique; ID100-50), coupled to a time-correlated single-photon counting (TCSPC) card (Becker \& Hickl GmbH; DPC-230). The atomic force microscope head is placed on top of the TAO stage and is operated in tapping (AC) mode. For scanning, we use Si-based Alcoated tips (NanoWorld NCHR-10 PointProbe) with $\sim 15 \mathrm{~nm}$ radius. The ideal lateral and vertical resolution of this system is $\sim 30$ and 0.1 $\mathrm{nm}$, respectively. The measured particles appear much broader in lateral dimensions than they are in reality because their lateral size and shape are given by the convolution of the tip size and shape and the particle's size and shape. Therefore, for convenience, the lateral data are typically not used or interpreted. The size of the scanning area is $20 \times 20 \mu \mathrm{m}^{2}$, which is divided into $2048 \times 2048$ steps in-plane and measured with a slow $0.5 \mathrm{~Hz}$ scanning rate. The alignment protocol is described in detail in the Supporting Information.

The measurement protocol for the correlated AFM and optical microspectroscopy is illustrated in Figure $2 \mathrm{~b}$,c. First, we construct the overall AFM images of the optical field of view (FOV) by stitching separate AFM scan patches. The correlation between the PL image and the AFM scan is done optically using the coarse alignment described in the previous paragraph. For the spectral resolution, a slit of $2 \mu \mathrm{m}$ width is introduced into the focal plane. The projection of the slit can be visualized in the PL image for precise control. This allows us to measure spectra of all of the Si-NPs falling into the slit at once. To cover the whole PL image, the slit is effectively scanned over the sample, which in practice is realized using the sample TAO stage with an accuracy of $0.1 \mu \mathrm{m} / \mathrm{step}$. To increase the photon counts, we bin the spectral signal by 8 pixels on the spectral axis, which is possible due to the broad spectral feature observed at room temperature. Also, we use spatial binning by 2 pixels. PL spectra are acquired for $6 \mathrm{~min}$. Subsequently, the background signal is removed by subtracting the signal from the neighboring (binned) pixels. The acquired PL emission rate of a typical single $\mathrm{Si}-\mathrm{NP}$ after subtracting the background is typically above 10 counts/s in a spectral bin. Finally, all spectra are corrected for the spectral response of the detection chain by use of a calibrated black-body-radiation source (tungsten halogen lamp and deuterium lamp). The correction curve (in the form of a multiplication correction factor) is shown in Figure S7. Spectra are fitted on the energy scale (after multiplying the intensity by the square of the wavelength to correct for nonlinear conversion between wavelength and energy) by a single Gaussian function (assuming inhomogeneous line broadening). ${ }^{57}$ Thanks to the direct correlation between the optical image and AFM, we can then correlate the size evaluated from the height of the NPs in the AFM scan and the optical band-gap energy, obtained from the Gaussian fit of the same single-dot PL spectrum as the peak energy.

Ensemble samples were also analyzed using standard UV-vis spectroscopy. Extinction spectra were measured using a standard 
spectrophotometer (PerkinElmer; Lambda 950) equipped with deuterium and tungsten halogen excitation lamps and a photomultiplier tube (PMT) detector, sensitive in the spectral range of $250-860 \mathrm{~nm}(1.442-4.959 \mathrm{eV})$. The system is equipped with a dualstage, which allowed us to simultaneously measure the sample and the reference. PL excitation (PLE) spectra of the ensemble were measured using a spectrofluorometer (Fluorolog-3; Horiba Scientific) equipped with a $450 \mathrm{~W}$ excitation xenon lamp (Ushio) and a Si-based CCD detector (Synapse; Horiba Scientific) in the VIS spectral range. Samples were excited between $300(4.133 \mathrm{eV})$ and $500 \mathrm{~nm}(2.480$ $\mathrm{eV})$ with a resolution of $5 \mathrm{~nm}$. All spectra were corrected for the spectral response of the detection system.

Samples were further analyzed by STEM (FEI Verios 460), microRaman spectroscopy (Witec- $\alpha 300$ Raman system; UHT S300S VIS spectroscopy, $532.3 \mathrm{~nm}$ excitation, Zeiss EC Epiplan-Neofluar 100× objective with a numerical aperture of 0.9 ), and $2 \theta \mathrm{XRD}$ spectrometry from 10 to $90^{\circ}$ with a $0.5^{\circ} / \mathrm{min}$ scanning rate (Rigaku; MiniFlex-II).

\section{ASSOCIATED CONTENT}

\section{(s) Supporting Information}

The Supporting Information is available free of charge at https://pubs.acs.org/doi/10.1021/acsanm.0c02395.

Description of the setup methodology of the correlative optical microspectroscopy and AFM; additional spectroscopy data (extinction, PL and PLE spectra of the sample, analysis of the Tauc plot for absorption of the sample; PLE map for a broad range of PL energies; single-dot PL spectra fitted by a Gaussian peak function; histogram of single-dot PL spectra FWHM; timedependent PL signal; and PL correction curve); and size estimation (topography of an empty substrate; size histogram of the Si-NPs obtained from STEM; and additional Raman spectra for Si-NP clusters) (PDF)

\section{AUTHOR INFORMATION}

\section{Corresponding Authors}

Chia-Ching Huang - Institute of Physics, University of Amsterdam, 1098 XH Amsterdam, The Netherlands;

Email: aching0901@gmail.com

Kateřina Dohnalová - Institute of Physics, University of Amsterdam, 1098 XH Amsterdam, The Netherlands; ๑ orcid.org/0000-0002-7333-6889; Email: k.newell@ uva.nl

\section{Authors}

Yingying Tang - Institute of Physics, University of Amsterdam, 1098 XH Amsterdam, The Netherlands

Marco van der Laan - Institute of Physics, University of Amsterdam, 1098 XH Amsterdam, The Netherlands

Jorik van de Groep - Institute of Physics, University of Amsterdam, 1098 XH Amsterdam, The Netherlands; (1) orcid.org/0000-0003-3033-8005

A. Femius Koenderink - Institute of Physics, University of Amsterdam, 1098 XH Amsterdam, The Netherlands; Center for Nanophotonics, AMOLF, 1098 XG Amsterdam, The Netherlands; @ orcid.org/0000-0003-1617-5748

Complete contact information is available at:

https://pubs.acs.org/10.1021/acsanm.0c02395

\section{Author Contributions}

C.-C.H. performed most of the measurements and data analysis. K.D. conceived and supervised the project. Y.T. provided XRD spectra and NP size statistics from STEM images. M.v.d.L. provided micro-Raman spectroscopy data on the setup facilitated by J.v.d.G. All authors provided feedback and contributed to the manuscript.

Notes

The authors declare no competing financial interest.

\section{ACKNOWLEDGMENTS}

This work is financially supported by NWO Projectruimte. SiNP samples were synthesized by S. Regli from the group of J.G.C. Veinot from the University of Alberta. C.-C.H. acknowledges the support of XRD facilities from van't Hoff Institute for Molecular Sciences (HIMS).

\section{REFERENCES}

(1) Canham, L. Introductory lecture: origins and applications of efficient visible photoluminescence from silicon-based nanostructures. Faraday Discuss. 2020, 222, 10-81.

(2) Dohnalová, K.; Gregorkiewicz, T.; Ko̊sová, K. Silicon quantum dots: surface matters. J. Phys.: Condens. Matter 2014, 26, No. 173201.

(3) Dohnalová, K.; Hapala, P.; Kůsová, K.; Infante, I. Electronic structure engineering achieved via organic ligands in silicon nanocrystals. Chem. Mater. 2020, 32, 6326-6337.

(4) Delley, B.; Steigmeier, E. F. Quantum confinement in Si nanocrystals. Phys. Rev. B 1993, 47, 1397-1400.

(5) Wang, X.; Huang, D.; Ye, L.; Yang, M.; Hao, P.; Fu, H.; Hou, X.; Xie, X. Pinning of photoluminescence peak positions for lightemitting porous silicon: An evidence of quantum size effect. Phys. Rev. Lett. 1993, 71, 1265-1267.

(6) Wolkin, M. V.; Jorne, J.; Fauchet, P. M.; Allan, G.; Delerue, C. Electronic States and Luminescence in Porous Silicon Quantum Dots: The Role of Oxygen. Phys. Rev. Lett. 1999, 82, 197-200.

(7) Carroll, G. M.; Limpens, R.; Neale, N. R. Tuning Confinement in Colloidal Silicon Nanocrystals with Saturated Surface Ligands. Nano Lett. 2018, 18, 3118-3124.

(8) Ghosh, B.; Shirahata, N. Colloidal silicon quantum dots: synthesis and luminescence tuning from the near-UV to the near-IR range. Sci. Technol. Adv. Mater. 2014, 15, No. 014207.

(9) Ni, Z.; Zhou, S.; Zhao, S.; Peng, W.; Yang, D.; Pi, X. Silicon nanocrystals: unfading silicon materials for optoelectronics. Mater. Sci. Eng., $R$ 2019, 138, 85-117.

(10) Brus, L. E. Electron-electron and electron-hole interactions in small semiconductor crystallites: The size dependence of the lowest excited electronic state. J. Chem. Phys. 1984, 80, 4403-4409.

(11) Ögüt, S.; Chelikowsky, J. R.; Louie, S. G. Quantum Confinement and Optical Gaps in Si Nanocrystals. Phys. Rev. Lett. 1997, 79, 1770-1773.

(12) Weissker, H.-C.; Furthmüller, J.; Bechstedt, F. Optical properties of $\mathrm{Ge}$ and $\mathrm{Si}$ nanocrystallites from $\mathrm{ab}$ initio calculations. I. Embedded nanocrystallites. Phys. Rev. B 2002, 65, No. 155327.

(13) Kůsovaá, K.; Ondič, L.; Klimešová, E.; Herynková, K.; Pelant, I.; Daniš, S.; Valenta, J.; Gallart, M.; Ziegler, M.; Hönerlage, B.; Gilliot, P. Luminescence of free-standing versus matrix-embedded oxide-passivated silicon nanocrystals: The role of matrix-induced strain. Appl. Phys. Lett. 2012, 101, No. 143101.

(14) Kủsová, K.; Hapala, P.; Valenta, J.; Jelínek, P.; Cibulka, O.; Ondič, L.; Pelant, I. Direct Bandgap Silicon: Tensile-Strained Silicon Nanocrystals. Adv. Mater. Interfaces 2014, 1, No. 1300042.

(15) Kůsová, K.; Cibulka, O.; Dohnalová, K.; Pelant, I.; Valenta, J.; Fučíková, A.; Źídek, K.; Lang, J.; Englich, J.; Matějka, P.; Stěpánek, P.; Bakardjieva, S. Brightly Luminescent Organically Capped Silicon Nanocrystals Fabricated at Room Temperature and Atmospheric Pressure. ACS Nano 2010, 4, 4495-4505.

(16) Thiessen, A. N.; Ha, M.; Hooper, R. W.; Yu, H.; Oliynyk, A. O.; Veinot, J. G. C.; Michaelis, V. K. Silicon Nanoparticles: Are They Crystalline from the Core to the Surface? Chem. Mater. 2019, 31, 678-688.

(17) Thiessen, A.; Zhang, L.; Oliynyk, A.; Yu, H.; O’Connor, K.; Meldrum, A.; Veinot, J. A Tale of Seemingly "Identical" Silicon 
Quantum Dot Families: Structural Insight into Silicon Quantum Dot Photoluminescence. Chem. Mater. 2020, 32, 6838-6846.

(18) Regli, S.; Kelly, J. A.; Shukaliak, A. M.; Veinot, J. G. C. Photothermal Response of Photoluminescent Silicon Nanocrystals. J. Phys. Chem. Lett. 2012, 3, 1793-1797.

(19) Van Dam, B.; Bruhn, B.; Kondapaneni, I.; Dohnal, G.; Wilkie, A.; Křivánek, J.; Valenta, J.; Mudde, Y.; Schall, P.; Dohnalová, K. Quantum Yield Bias in Materials With Lower Absorptance. Phys. Rev. Appl. 2019, 12, No. 024022.

(20) Sinelnikov, R.; Dasog, M.; Beamish, J.; Meldrum, A.; Veinot, J. G. C. Revisiting an Ongoing Debate: What Role Do Surface Groups Play in Silicon Nanocrystal Photoluminescence. ACS Photonics 2017, 4, 1920-1929.

(21) Sychugov, I.; Valenta, J.; Linnros, J. Probing silicon quantum dots by single-dot techniques. Nanotechnology 2017, 28, No. 072002.

(22) Vasiliev, I.; Ögüt, S.; Chelikowsky, J. R. Ab Initio Absorption Spectra and Optical Gaps in Nanocrystalline Silicon. Phys. Rev. Lett. 2001, 86, 1813-1816.

(23) de Boer, W. D. A. M.; Timmerman, D.; Dohnalova, K.; Yassievich, I. N.; Zhang, H.; Buma, W. J.; Gregorkiewicz, T. Red spectral shift and enhanced quantum efficiency in phonon-free photoluminescence from silicon nanocrystals. Nat. Nanotechnol. 2010, $5,878-884$.

(24) Puzder, A.; Williamson, A. J.; Grossman, J. C.; Galli, G. Surface Chemistry of Silicon Nanoclusters. Phys. Rev. Lett. 2002, 88, No. 097401.

(25) Kůsová, K.; Pelant, I.; Valenta, J. Bright trions in direct-bandgap silicon nanocrystals revealed by low-temperature single-nanocrystal spectroscopy. Light: Sci. Appl. 2015, 4, No. e336.

(26) Sychugov, I.; Fucikova, A.; Pevere, F.; Yang, Z.; Veinot, J. G. C.; Linnros, J. Ultranarrow Luminescence Linewidth of Silicon Nanocrystals and Influence of Matrix. ACS Photonics 2014, 1, 998-1005.

(27) Wang, L. W.; Zunger, A. Electronic Structure Pseudopotential Calculations of Large (.apprx.1000 Atoms) Si Quantum Dots. J. Phys. Chem. A. 1994, 98, 2158-2165.

(28) Delerue, C.; Allan, G.; Lannoo, M. Theoretical aspects of the luminescence of porous silicon. Phys. Rev. B 1993, 48, 11024-11036.

(29) Takeoka, S.; Fujii, M.; Hayashi, S. Size-dependent photoluminescence from surface-oxidized $\mathrm{Si}$ nanocrystals in a weak confinement regime. Phys. Rev. B 2000, 62, 16820-16825.

(30) Ledoux, G.; Gong, J.; Huisken, F.; Guillois, O.; Reynaud, C. Photoluminescence of size-separated silicon nanocrystals: Confirmation of quantum confinement. Appl. Phys. Lett. 2002, 80, 4834-4836.

(31) Kim, T.-W.; Cho, C.-H.; Kim, B.-H.; Park, S.-J. Quantum confinement effect in crystalline silicon quantum dots in silicon nitride grown using SiH4 and NH3. Appl. Phys. Lett. 2006, 88, No. 123102.

(32) Park, N.-M.; Choi, C.-J.; Seong, T.-Y.; Park, S.-J. Quantum Confinement in Amorphous Silicon Quantum Dots Embedded in Silicon Nitride. Phys. Rev. Lett. 2001, 86, 1355-1357.

(33) Kim, C.-H.; Choi, J.; Bonnassieux, Y.; Horowitz, G. Simplified Numerical Simulation of Organic Photovoltaic Devices. J. Comput. Electron. 2016, 15, 1095-1102.

(34) Jarolimek, K.; Hazrati, E.; de Groot, R. A.; de Wijs, G. A. Band Offsets at the Interface between Crystalline and Amorphous Silicon from First Principles. Phys. Rev. Appl. 2017, 8, No. 014026.

(35) Vasques, A.; Torchynska, T.; Polupan, G.; Matsumoto, Y.; Khomenkova, L.; Shcherbyna, L. Size Dependent Photoluminescence of Si Nano-Crystals Embedded in Amorphous Silicon. Solid State Phenom. 2008, 131-133, 71-76.

(36) Khoo, K. H.; Zayak, A. T.; Kwak, H.; Chelikowsky, J. R. FirstPrinciples Study of Confinement Effects on the Raman Spectra of Si Nanocrystals. Phys. Rev. Lett. 2010, 105, No. 115504.

(37) Gaisler, S. V.; Semenova, O. I.; Sharafutdinov, R. G.; Kolesov, B. A. Analysis of Raman spectra of amorphous-nanocrystalline silicon films. Phys. Solid State 2004, 46, 1528-1532.

(38) Waman, V.; Funde, A.; Kamble, M.; Rajanna, P.; Hawaldar, R.; Amalnerkar, D.; Sathe, V.; Gosavi, S.; Jadkar, S. Hydrogenated
Nanocrystalline Silicon Thin Films Prepared by Hot-Wire Method with Varied Process Pressure. J. Nanotechnol. 2011, 2011, No. 242398.

(39) Popovic, D. M.; Milosavljevic, V.; Zekic, A.; Romcevic, N.; Daniels, S. Raman scattering analysis of silicon dioxide single crystal treated by direct current plasma discharge. Appl. Phys. Lett. 2011, 98, No. 051503.

(40) Yadav, A. K.; Singh, P. A review of the structures of oxide glasses by Raman spectroscopy. RSC Adv. 2015, 5, 67583-67609.

(41) Zaumseil, P. High-resolution characterization of the forbidden Si 200 and Si 222 reflections. J. Appl. Crystallogr. 2015, 48, 528-532.

(42) Rossmanith, E. Approximate calculation of multiple-diffraction patterns based on Renninger's kinematical 'simplest approach. J. Appl. Crystallogr. 2000, 33, 921-927.

(43) Zhao, L.; Steinhart, M.; Yosef, M.; Lee, S. K.; Geppert, T.; Pippel, E.; Scholz, R.; Gösele, U.; Schlecht, S. Lithium Niobate Microtubes within Ordered Macroporous Silicon by Templated Thermolysis of a Single Source Precursor. Chem. Mater. 2005, 17, 35 .

(44) Hesse, D.; Lee, S. K.; Gösele, U. Microstructure of (104)oriented $\mathrm{Bi}_{3.25} \mathrm{La}_{0.75} \mathrm{Ti}_{3} \mathrm{O}_{12}$ and $\mathrm{Bi}_{3.54} \mathrm{Nd}_{0.46} \mathrm{Ti}_{3} \mathrm{O}_{12}$ ferroelectric thin films on multiply twinned $\mathrm{SrRuO}_{3} / \mathrm{Pt}(111)$ electrodes on $\mathrm{YSZ}(100)$ buffered Si(100). Phys. Status Solidi A 2005, 202, 2287-2298.

(45) Ebenstein, Y.; Nahun, E.; Banin, U. Tapping Mode Atomic Force Microscopy for Nanoparticle Sizing: Tip-Sample Interaction Effects. Nano Lett. 2002, 2, 945-950.

(46) Lu, J. R.; Thomas, R. K.; Binks, B. P.; Fletcher, P. D. I.; Penfold, J. Structure and Composition of Dodecane Layers Spread on Aqueous Solutions of Dodecyl- and Hexadecyltrimethylammonium Bromides Studied by Neutron Reflection. J. Phys. Chem. B. 1995, 99, 41134123.

(47) Kim, B.-s.; Kang, H. J.; Seo, S.; Park, N. S. Effect of Dodecane on the Surface Structure and the Electronic Properties of Pentacene on Modified Si (001). Appl. Sci. Convergence Technol. 2016, 25, 2831.

(48) Dohnalová, K.; Fučíková, A.; Umesh, C. P.; Humpolícková, J.; Paulusse, J. M. J.; Valenta, J.; Zuilhof, H.; Hof, M.; Gregorkiewicz, T. Microscopic Origin of the Fast Blue-Green Luminescence of Chemically Synthesized Non-oxidized Silicon Quantum Dots. Small 2012, 8, 3185-3191.

(49) van Dam, B.; Nie, H.; Ju, B.; Marino, E.; Paulusse, J. M. J.; Schall, P.; Li, M.; Dohnalová, K. Excitation-Dependent Photoluminescence from Single-Carbon Dots. Small 2017, 13, No. 1702098

(50) Alessi, B.; Macias-Montero, M.; Maddi, C.; Maguire, P.; Svrcek, V.; Mariotti, D. Bridging energy bands to the crystalline and amorphous states of Si QDs. Faraday Discuss. 2020, 390-404.

(51) Talyzin, I. V.; Samsonov, M. V.; Samsonov, V. M.; Pushkar, M. Y.; Dronnikov, V. V. Size Dependence of the Melting Point of Silicon Nanoparticles: Molecular Dynamics and Thermodynamic Simulation. Semiconductors 2019, 53, 947-953.

(52) Thomson, W. LX. On the equilibrium of vapour at a curved surface of liquid. London, Edinburgh Dublin Philos. Mag. J. Sci. 1871, $42,448-452$.

(53) Goldstein, A. N.; Echer, C. M.; Alivisatos, A. P. Melting in Semiconductor Nanocrystals. Science 1992, 256, 1425-1427.

(54) Wautelet, M. Estimation of the variation of the melting temperature with the size of small particles, on the basis of a surfacephonon instability model. J. Phys. D: Appl. Phys. 1991, 24, 343-346.

(55) Goldstein, A. The melting of silicon nanocrystals: Submicron thin-film structures derived from nanocrystal precursors. Appl. Phys. A: Mater. Sci. Process. 1996, 62, 33-37.

(56) Hirasawa, M.; Orii, T.; Seto, T. Size-dependent crystallization of Si nanoparticles. Appl. Phys. Lett. 2006, 88, No. 093119.

(57) Würth, C.; Grabolle, M.; Pauli, J.; Spieles, M.; Resch-Genger, $\mathrm{U}$. Relative and absolute determination of fluorescence quantum yields of transparent samples. Nat. Protoc. 2013, 8, 1535-1550. 